\title{
Geometry of duskside equatorial current during magnetic storm main phase as deduced from magnetospheric and low-altitude observations
}

\author{
S. Dubyagin ${ }^{1}$, N. Ganushkina ${ }^{1,2}$, S. Apatenkov ${ }^{3}$, M. Kubyshkina ${ }^{3}$, H. Singer ${ }^{4}$, and M. Liemohn ${ }^{2}$ \\ ${ }^{1}$ Finnish Meteorological Institute, Erik Palmenin aukio 1, Helsinki, 00101, Finland \\ ${ }^{2}$ Department of Atmospheric, Oceanic and Space Sciences, University of Michigan, 2455 Hayward St., Ann Arbor, MI \\ 48109-2143, USA \\ ${ }^{3}$ St. Petersburg State University, Earth Physics Department, Ulyanovskaya 1, Petrodvoretz, St. Petersburg, 198504, Russia \\ ${ }^{4}$ Space Weather Prediction Center, 325 Broadway, Boulder, CO, USA \\ Correspondence to: S. Dubyagin (stepan.dubyagin@ fmi.fi)
}

Received: 17 October 2012 - Revised: 16 January 2013 - Accepted: 8 February 2013 - Published: 4 March 2013

\begin{abstract}
We present the results of a coordinated study of the moderate magnetic storm on 22 July 2009. The THEMIS and GOES observations of magnetic field in the inner magnetosphere were complemented by energetic particle observations at low altitude by the six NOAA POES satellites. Observations in the vicinity of geosynchronous orbit revealed a relatively thin (half-thickness of less than $1 R_{\mathrm{E}}$ ) and intense current sheet in the dusk MLT sector during the main phase of the storm. The total westward current (integrated along the z-direction) on the duskside at $r \sim 6.6 R_{\mathrm{E}}$ was comparable to that in the midnight sector. Such a configuration cannot be adequately described by existing magnetic field models with predefined current systems (error in $B>60 \mathrm{nT}$ ). At the same time, low-altitude isotropic boundaries (IB) of $>80 \mathrm{keV}$ protons in the dusk sector were shifted $\sim 4^{\circ}$ equatorward relative to the IBs in the midnight sector. Both the equatorward IB shift and the current strength on the duskside correlate with the Sym- $\mathrm{H}^{*}$ index. These findings imply a close relation between the current intensification and equatorward IB shift in the dusk sector. The analysis of IB dispersion revealed that high-energy IBs $(E>100 \mathrm{keV})$ always exhibit normal dispersion (i.e., that for pitch angle scattering on curved field lines). Anomalous dispersion is sometimes observed in the low-energy channels $(\sim 30-100 \mathrm{keV})$. The maximum occurrence rate of anomalous dispersion was observed during the main phase of the storm in the dusk sector.
\end{abstract}

Keywords. Magnetospheric physics (Current systems; Energetic particles, precipitating; Storms and substorms)

\section{Introduction}

The dusk-dawn asymmetry of the magnetic field in the inner magnetosphere during storm times has been known for decades (Cahill Jr., 1966). Usually, it is attributed to development of a partial ring current (PRC) in the dusk-midnight sector (Cummings, 1966; Siscoe and Crooker, 1974; Crooker and Siscoe, 1981; Iijima et al., 1990; Nakabe et al., 1997; Liemohn et al., 2001a). Knowledge of the magnetic configuration on the eveningside is very important for space weather applications. Ions, which are the main mass and energy carriers in the magnetosphere, drift westward after an injection populating the ring current region and eventually influence the outer radiation belts. There is also evidence that the PRC can be the main contributor to ground magnetic field disturbances at low latitudes during the storm main phase (Liemohn et al., 2001b). Recent advances in the empirical modeling of the storm time magnetic configuration (Tsyganenko and Sitnov, 2007) as well as in the methods of statistical analysis (Le et al., 2004) have shown that the duskside current may exhibit significant deviations from the conventional shape.

Low-altitude particle observations can be a powerful tool for remote sensing of the equatorial magnetic field (e.g., 
Sergeev et al., 1993). These data have already been used for studying the storm time processes. For example, Hauge and Søraas (1975) noticed that the equatorial boundary of the proton precipitation is well related to the Dst index. In other studies (Søraas et al., 2002; Asikainen et al., 2010), the low-altitude particle observations were used to predict the Dst index. However, there are many factors which make the interpretation of these observations questionable, especially during storm time in the dusk sector. Among them are an uncertainty of the mapping between the equatorial magnetosphere and low altitudes, and an unknown mechanism of the equatorial isotropic boundary formation. Although there is strong evidence that scattering on curved field lines is the main mechanism of loss cone filling for protons on the nightside (see Sergeev et al., 1993, and references therein), it is not necessarily true during geomagentic storms. It is known that pitch angle scattering by electromagnetic ion cyclotron (EMIC) waves may produce isotropic or almost isotropic proton fluxes at low latitude (Søraas et al., 1980; Gvozdevsky et al., 1997; Yahnin and Yahnina, 2007, and references therein). Direct spacecraft measurements have shown that EMIC waves are abundant in the inner magnetosphere at dusk during storms (e.g., Bräysy et al., 1998; Halford et al., 2010 , and references therein).

Given the disparity of conclusions about the location and intensity of current systems in near-Earth space, in this paper we investigate this issue with a coordinated data model analysis study. We analyze a moderate storm on 22 July 2009 to determine the geometry of the current system in the dusk sector. The descriptions of the storm and spacecraft orbit configuration are given in Sect. 2. In Sect. 3, using THEMIS (Sibeck and Angelopoulos, 2008) and GOES spacecraft observations in the inner magnetosphere, we show that the duskside magnetic configuration in the inner magnetosphere is highly stretched during the storm main phase. In Sect. 4 we use the Tsyganenko and Sitnov (2005) model (hereafter TS05) to figure out whether the standard current systems can reproduce the observed magnetic field. The data of six lowaltitude NOAA POES satellites were also available during this storm, providing good MLT and temporal coverage on the storm time-scale. The in situ observations in the equatorial plane together with TS05 were used to estimate possible mapping inaccuracies. We also attempt to distinguish between the two loss cone filling mechanisms. For that purpose, we analyze the dispersion of isotropic boundaries for particles of different energies. These results are presented in Sect. 5. In Sect. 6 we discuss our results in light of previous statistical studies.

\section{Event description}

The magnetic storm on 22-23 July 2009 was caused by a high-speed stream and has been analyzed in a number of studies (see Ganushkina et al., 2012; Perez et al., 2012, and

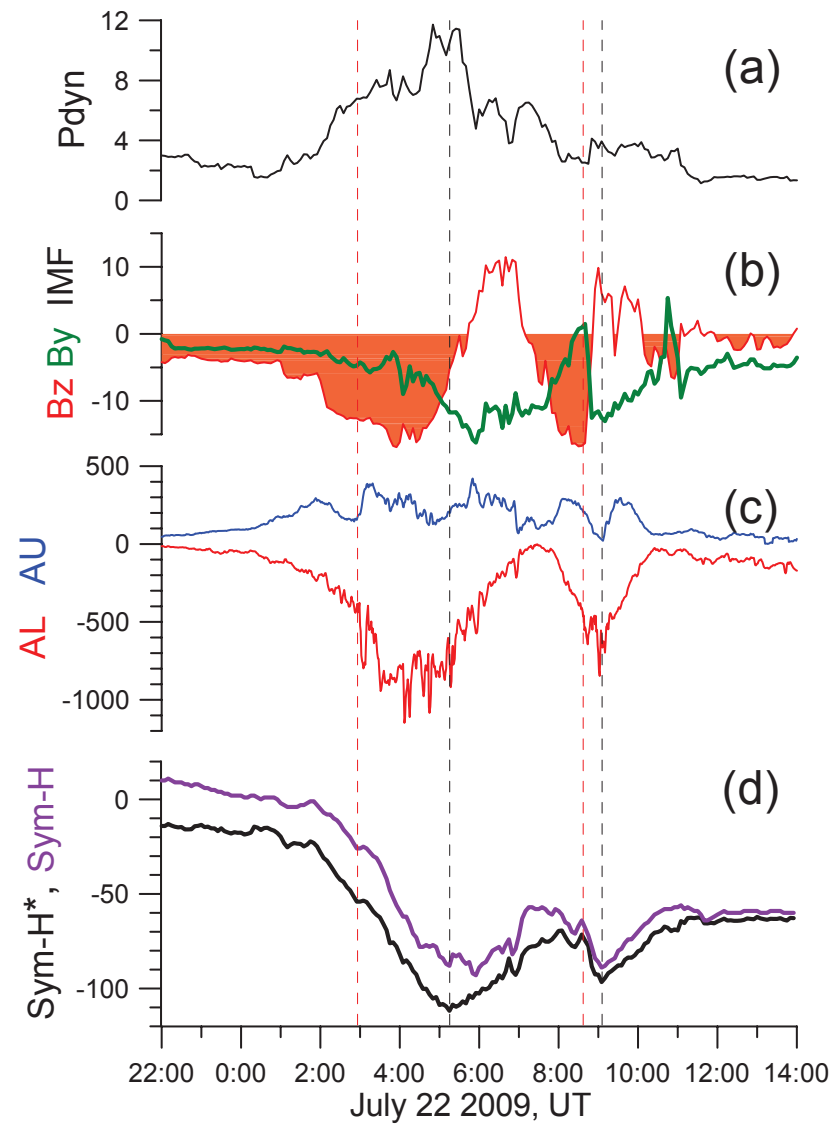

Fig. 1. Solar wind parameters and geomagnetic activity indices: (a) solar wind dynamic pressure in $\mathrm{nPa}$, (b) IMF $B_{\mathrm{z}}$ (red) and $B_{\mathrm{y}}$ (green) in nT, (c) AU and AL indices (blue and red curves respectively). (d) Sym-H index (magenta) and Sym-H* (black). The vertical dashed lines mark the beginning of rapid AL intensifications (red) and Sym-H* minima (black).

reference therein). Figure 1 shows solar wind parameters and the geomagnetic activity indices AU, AL, Sym-H and Sym$\mathrm{H}^{*}$. Sym- $\mathrm{H}^{*}$ is the Sym-H index with the Chapman-Ferraro and ground-induced currents contribution subtracted, Sym$\mathrm{H}^{*}=0.8$ Sym-H $-13 \sqrt{P_{\text {dyn }}}$ (e.g., Tsyganenko, 1996). For this storm, Sym- $\mathrm{H}^{*}$ has two minima $(-111 \mathrm{nT}$ and $-96 \mathrm{nT})$ at 05:15 and 09:05 UT of 22 July associated with two periods of strong negative IMF $B_{\mathrm{Z}}$. The AL index also has two distinct intensifications attaining $\sim-900$ and $-600 \mathrm{nT}$ at $\sim$ 04:00 and $\sim$ 09:00 UT. The beginnings of the AL intensifications are marked by red dashed lines and the Sym- $\mathrm{H}^{*}$ minima are marked by black dashed lines. Figure $2 \mathrm{a}$ and $\mathrm{b}$ show orbit segments during the two periods of Sym- $\mathrm{H}^{*}$ dip. Only the XY-projection is shown since all spacecraft are close to the equatorial plane with $Z_{\mathrm{GSM}}$ between -1.5 and $+2.5 R_{\mathrm{E}}$. The labels at the symbols show UT hours. Similar spacecraft configurations occurred during both $\mathrm{Sym}-\mathrm{H}^{*}$ decreases with at least one spacecraft in the dusk sector and another at local midnight. During the first decrease (02:00-05:00 UT, 

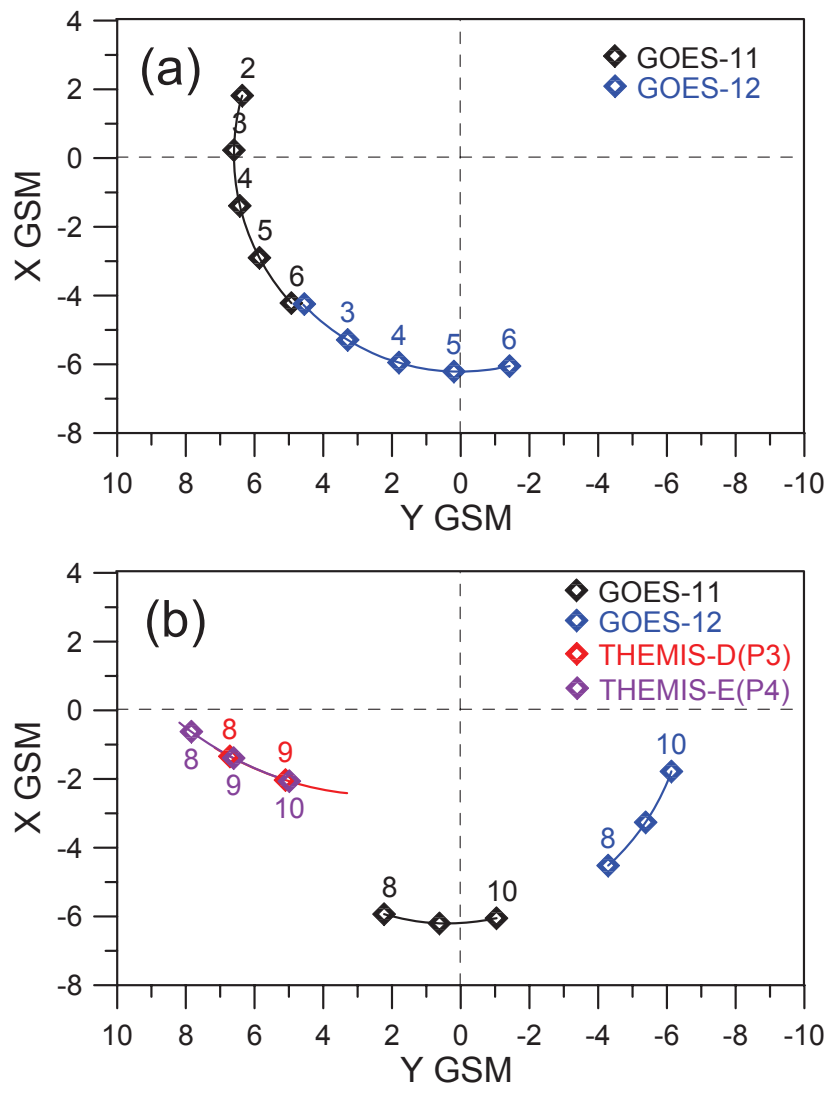

Fig. 2. Projection of spacecraft orbits on the XY GSM plane. (a) The first Sym-H* dip period (02:00-06:00 UT), (b) second Sym$\mathrm{H}^{*}$ dip period (08:00-10:00 UT). The colors correspond to different spacecraft.

Fig. 2a) GOES-11 entered the nightside from the dusk sector and GOES-12 moved from $\sim 21: 00$ MLT to midnight. During the second decrease (08:00-09:00 UT, Fig. 2b) THEMIS probes $\mathrm{D}(\mathrm{P} 3)$ and $\mathrm{E}(\mathrm{P} 4)$ successively passed inside geosynchronous orbit in the dusk sector 19:00 MLT when GOES11 and -12 were at $\sim$ 23:00 and $\sim$ 03:00 MLT, respectively.

\section{Magnetospheric observations}

Figure 3 shows observations from four spacecraft in the vicinity of geosynchronous orbit. To represent the magnetic field measurements, we use cylindrical GSM coordinates $(z, r, \varphi)$ with the $\mathrm{z}$-axis coinciding with $z$ GSM and the $\boldsymbol{r}$ unit vector is outward from the $\mathrm{z}$-axis and $\varphi$ is eastward. The three bottom panels in Fig. 3 show the $z, \varphi, r$ components of the external magnetic field (IGRF field has been subtracted). The black curve corresponds to the satellite observations and the red curves correspond to the models that will be discussed later. The blue curves in the top panels show the distance to the neutral sheet (NS) estimated from the Tsyganenko and Fairfield (2004) model. In the near-Earth region, the current sheet undergoes fewer flapping oscillations and the model estimation of this parameter is expected to be realistic.

During the first Sym- $\mathrm{H}^{*}$ dip at 02:00-05:15 UT, the magnetic field at geosynchronous orbit exhibits signatures of an equatorial current enhancement: strengthening of $\left|B_{\mathrm{r}}\right|$ and decrease of $B_{\mathrm{Z}}$. In the premidnight sector, this enhancement is briefly interrupted by a dipolarization observed by GOES12 (Fig. 3b) at the beginning of the first AL intensification (marked by a red dashed vertical line). At approximately the same time, the radial component of the external field at GOES-11 (Fig. 3a) in the 18:00-20:00 MLT sector starts to decrease and attains a value of $-120 \mathrm{nT}$. The full field components (dipole field not subtracted - not shown) observed by GOES-11 at 05:30 UT (the moment of minimum $B_{\mathrm{r}}$ ) were $B_{\mathrm{r}}^{\text {full }}=-153$ and $B_{\mathrm{z}}^{\text {full }}=17 \mathrm{nT}$, indicating that the duskside magnetic field had an extremely stretched configuration at the geosynchronous location. Using Maxwell's equation, $\mu_{0} j=\nabla \times \boldsymbol{B}$, and assuming that $\partial B_{\mathrm{Z}} / \partial r<<\partial B_{\mathrm{r}} / \partial z$, which is valid for stretched configurations, we can roughly estimate the total azimuthal current integrated between $+Z_{\mathrm{SC}}$ and $-Z_{\mathrm{SC}} ; I=\left|\int j_{\varphi} d z\right| \approx 2\left|B_{\mathrm{r}}\left(Z_{\mathrm{SC}}\right)\right| / \mu_{0}$. Here $Z_{\mathrm{SC}}$ is the coordinate of the spacecraft position. The black curves in the top panels of Fig. 3 show $I=2\left|B_{\mathrm{r}}\right| / \mu_{0}$, which is the estimate of total equatorial current per $1 R_{\mathrm{E}}$ of radial distance. During the first Sym-H* dip, GOES-11 and -12 were $\sim 0.8 R_{\mathrm{E}}$ and $\sim 1.4 R_{\mathrm{E}}$ above the model NS, respectively. As it can be seen in Fig. 3a and b, maximal I values at 20:00 MLT (GOES-11) are higher than at midnight (GOES-12), although GOES-11 is located closer to NS than GOES-12. Since the current sheet in the midnight sector is expected to be thin during the main phase (the TS05 model gives a half-thickness $<1 R_{\mathrm{E}}$ ) and both the duskside and midnight I values are $\sim 1 M A / R_{\mathrm{E}}$, we conclude that all current on the duskside flows between $-Z_{\mathrm{SC}}$ and $+Z_{\mathrm{SC}}$, implying a current sheet half-thickness $\leq 1 R_{\mathrm{E}}$.

Similar signatures are seen during the second Sym- $\mathrm{H}^{*}$ dip. The THEMIS probes (Fig. 3c, d) were at $\sim$ 19:00 MLT and $\left|d Z_{\mathrm{NS}}\right|<0.8 R_{\mathrm{E}}$, closer to the NS than the GOES spacecraft were during the first $\mathrm{Sym}-\mathrm{H}^{*}$ dip. Again the observations show strengthening of $\left|B_{\mathrm{r}}\right|$ and decrease of $B_{\mathrm{z}}$. The full magnetic field components were $B_{\mathrm{r}}^{\text {full }}=126 \mathrm{nT}, B_{\mathrm{z}}^{\text {full }}=$ $65 \mathrm{nT}$ (THEMIS D(P3) at $r=5.2 R_{\mathrm{E}}$ ) and $B_{\mathrm{r}}^{\text {full }}=122 \mathrm{nT}$, $B_{\mathrm{Z}}^{\text {full }}=14 \mathrm{nT}$ (THEMIS E(P4) at $r=6.6 R_{\mathrm{E}}$ ) at that time. The leading probe, $\mathrm{P} 3$, observes stronger $B_{\mathrm{Z}}^{\text {full }}$ than the $\mathrm{P} 4$ probe; however, magnetic inclination is still $<30^{\circ}$, indicating that P3 is in the transition region between the tail-like and dipole-like configuration. Around that time GOES-11 (Fig. 3a) was at local midnight $\sim 0.9 R_{\mathrm{E}}$ above the NS and observed signatures of dipolarizations. Comparing the maximum total current values at the duskside (Fig. 3d top panel) and values at local midnight preceding the dipolarization during 08:00-09:00 UT (Fig. 3a top panel), we again find comparable values of $I \sim 0.8 M A / R_{\mathrm{E}}$. Note that the azimuthal component of the magnetic field is rather small, indicating that the current flows in the azimuthal direction so that the 

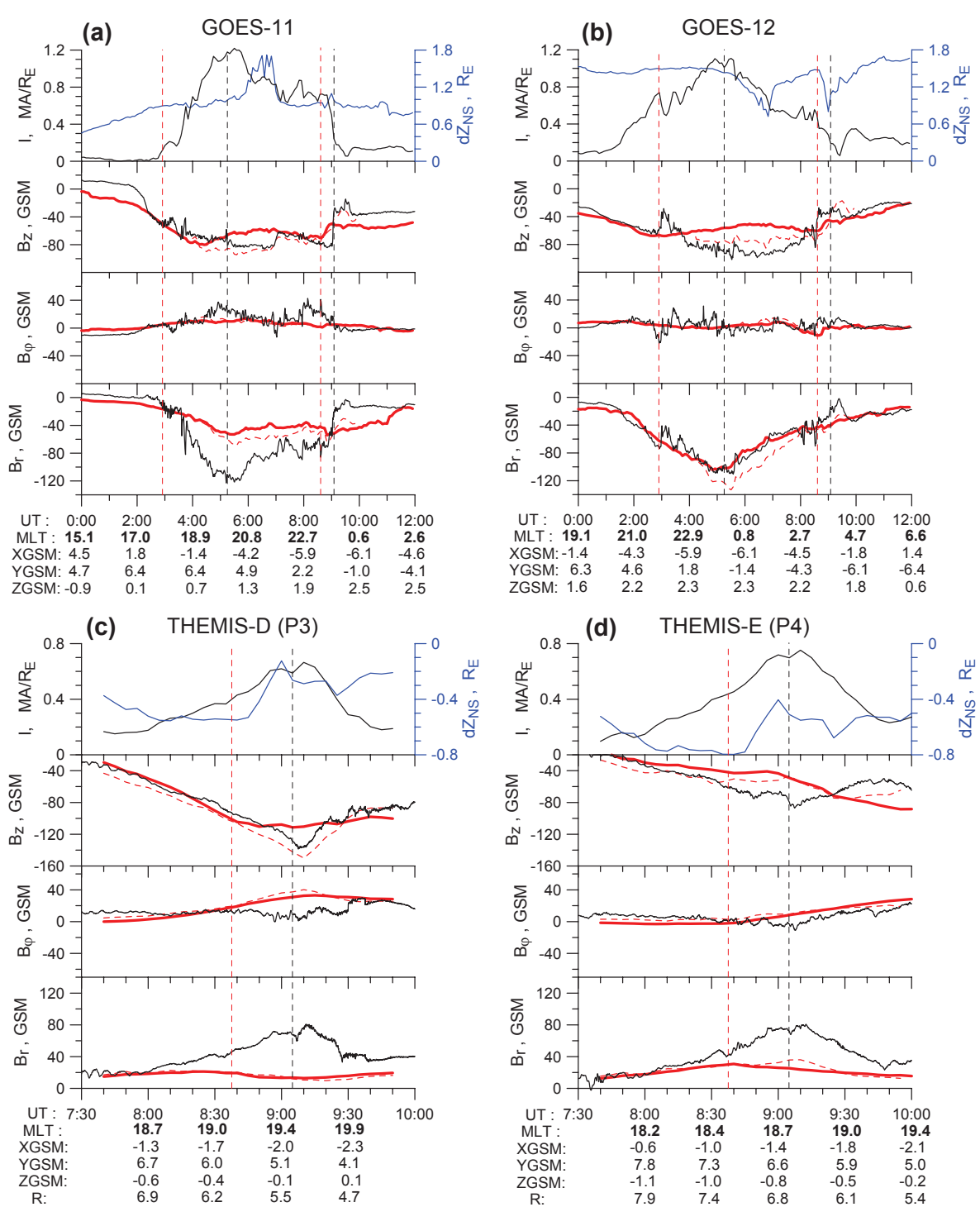

Fig. 3. (a)-(d) Observations of four spacecraft. Top panel: the estimate of the total current $I=2 B_{\mathrm{r}} / \mu_{0}$ in units of $M A / R_{\mathrm{E}}$ (black curve) and distance to the model neutral sheet (blue curve). The next three panels show the $B_{\mathrm{Z}}, B_{\varphi}$, and $B_{\mathrm{r}}$ components (dipole field subtracted) in nT: black - spacecraft observations; red solid curve - TS05 model field; red dashed curve - TS05MOD field. Red and black vertical lines mark beginnings of rapid AL intensifications and Sym- $\mathrm{H}^{*}$ minima, respectively.

total current values estimated at the same radial distance can be compared.

\section{Modeling results}

It is important to check whether the observed magnetic field signature can be described in terms of classical storm time current systems (PRC, tail current, etc) or if it is a manifestation of some unknown current. We compare the observations with TS05, a model that comprises all current systems and has been shown to be a good choice for modeling this particular storm (Ganushkina et al., 2012). The red solid curves in Fig. 3 represent the model field components. The largest deviations of the TS05 field from observations (as large as $\sim 50-60 \mathrm{nT}$ ) are seen in $B_{\mathrm{r}}$ on the duskside during both Sym$\mathrm{H}^{*}$ minima periods (Fig. 3a, c). The difference in $B_{\mathrm{Z}}$ and $B_{\varphi}$ is also significant although less than $30 \mathrm{nT}$. To check whether it is possible to describe the observed signatures using the TS05 PRC module, we modified the model by introducing three variable parameters: a multiplier to the PRC module field strength, an angle of the PRC rotation in the equatorial plane, and a multiplier to the tail mode 2 module field (see Tsyganenko and Sitnov, 2005, for TS05 module descriptions). The first two parameters are used to improve the representation of the field in the duskside sector, and the last one is introduced to improve $B_{\mathrm{Z}}$ at local midnight (tail mode 2 
current basically flows outside $r=6.6 R_{\mathrm{E}}$ and contributes mostly to $B_{\mathrm{Z}}$ at geosynchronous distance (Ganushkina et al., 2012)). We varied these parameters to minimize the error between the model and spacecraft observations in a manner described in Ganushkina et al. (2012). The resulting field components of this modified TS05 model (hereafter TS05MOD) are shown in Fig. 3 by the red dashed lines. From visual inspection it is clear that this only provides a minor improvement of the magnetic field on the duskside in comparison with the TS05 values. TS05MOD can describe the variation in $B_{\mathrm{Z}}$ but fails to describe the strong $B_{\mathrm{r}}$ on the duskside. An inverse problem for a model having three free parameters and matching two/three point vector measurements concentrated at a similar radial distance is likely ill-posed (under-defined). This is why we do not give the resulting parameter values, but rather concentrate on the resulting field. The goal of this modeling is to show that it is impossible to describe the existing configuration with the TS05 current systems without significant changes of their geometry.

\section{Low-altitude observations}

\subsection{Isotropic boundaries}

An additional information about the magnetic configuration and the processes in the equatorial plane can be obtained from low-altitude observations of the isotropic boundary (IB). This boundary separates regions of adiabatic and chaotic regimes of particle motions in the equatorial current sheet. It also separates regions of particle distribution having empty and filled loss cones and can be determined from low-altitude particle observations. Numerical simulations have shown that pitch angle scattering fills the loss cone at $R_{\mathrm{c}} / \rho \leq 8$ (e.g., Sergeev and Tsyganenko, 1982). Here $R_{\mathrm{c}}$ is the minimum field line curvature radius and $\rho$ is maximum particle gyroradius (see Sergeev et al. (1993) and references therein for more details about IBs). Using this criterion, the position of the IB can be determined from magnetic field models, assuming that there is no other scattering mechanism acting in this region. This ratio can be expanded as $R_{\mathrm{c}} / \rho \sim B_{\mathrm{z}}^{2} /\left(\partial B_{\mathrm{r}} / \partial z\right) \approx B_{\mathrm{z}}^{2} /\left(\mu_{0} j\right)$. It shows that the latitude of the IB is highly sensitive to the magnitude of the normal component in the current sheet. The strength of the $B_{\mathrm{Z}}$ depression is a rough measure of how much westward current flows outside of the observation point. During an equatorial current intensification, a region of strong scattering extends toward Earth and IB moves equatorward. For that reason the IB latitude can be used as an indicator of total current strength if there is no other scattering mechanism acting.

Although unambiguous determination of the type of the isotropization mechanism from low-altitude observations is barely possible, valuable information can be obtained from analysis of IBs for particles of different energy. If the IB lo-
Table 1. Estimated low-energy thresholds for proton $0^{\circ}$ detectors (according to Asikainen et al., 2012).

\begin{tabular}{lccc}
\hline Sat. name & P1 & P2 & P3 \\
\hline NOAA-15 & $64 \mathrm{keV}$ & $187 \mathrm{keV}$ & $415 \mathrm{keV}$ \\
NOAA-16 & $45 \mathrm{keV}$ & $140 \mathrm{keV}$ & $434 \mathrm{keV}$ \\
NOAA-17 & $45 \mathrm{keV}$ & $121 \mathrm{keV}$ & $374 \mathrm{keV}$ \\
NOAA-18 & $30 \mathrm{keV}$ & $93 \mathrm{keV}$ & $266 \mathrm{keV}$ \\
METOP & $36 \mathrm{keV}$ & $92 \mathrm{keV}$ & $271 \mathrm{keV}$ \\
\hline
\end{tabular}

cation is controlled by $R_{\mathrm{c}} / \rho$ parameter, the IBs of particles having different energy must exhibit dispersion because gyroradius depends on particle energy. The higher the energy, the lower the latitude of the boundary. The opposite order of IBs is usually interpreted as indication that IBs are formed by wave-particle interactions (e.g., Sergeev et al., 2010). In Sect. 5.3, we analyze the proton energy dispersions to conclude when and where the usage of $R_{\mathrm{c}} / \rho=8$ criterion for IB can be justified and we present the comparison of the observed and model IBs in Sect. 5.4.

\subsection{NOAA/POES particle data}

The data of Medium Energy Proton and Electron Detector (MEPED, Evans and Greer, 2000) on board the National Oceanic and Atmospheric Administration Polar Orbiting Environment Satellites (NOAA/POES) were used to determine IB locations. NOAA/POES satellites (hereafter NOAA) have nearly-circular orbits with altitude $\sim 850 \mathrm{~km}$ and orbital period $\sim 100$ min crossing the auroral oval four times per orbit. Six satellites - METOP, NOAA-15, 16, 17, 18 and 19 - covered most of the nightside MLT sector of the auroral oval during the period 00:00-12:00 UT of 22 July 2009. The MEPED detector has two telescopes measuring fluxes of trapped particles and those precipitating into the loss cone allowing IB determination. A telescope measuring fluxes of precipitating particles is referred to as " $0^{\circ}$ detector", whereas one measuring trapped flux is referred as " $90^{\circ}$ detector". The notations $F^{0}$ and $F^{90}$ are used to denote the corresponding flux values. The fluxes are measured in a few energy bands for ions, which are assumed to be protons, and for electrons. We use data from the first three proton energy bands, referred to as P1 (30-80 keV), P2 (80-240 keV), P3 (240-800 keV). Unfortunately, the MEPED detectors are subjected to radiation damage. The impact of this damage increases the energy threshold from their nominal level (e.g., Asikainen et al., 2012). The $90^{\circ}$ detector degrades faster than $0^{\circ}$ detector and it is impossible to determine IB location from the raw data without additional calibration after a few years of satellite operation. We have elaborated the calibration procedure for the measured $90^{\circ}$ flux which normalizes its value to the $0^{\circ}$-detector energy range. This procedure is described in Appendix A. Although after such correction IB can be reliably determined (within a certain accuracy which is discussed 
later), this IB cannot be referred to the nominal energy because of the shift of the $0^{\circ}$-detector low-energy limit. These low-energy limits estimated according to Asikainen et al. (2012) for the year 2009 are given in Table 1. It can be seen that the P1, P2, P3 energy bands always keep their order (P1 - lowest energy; P3 - highest), however, it should be kept in mind that their real energies can be significantly different from the nominal ones, especially for NOAA-15.

An analysis of IB energy dispersion requires a high accuracy of determination of IB location because latitudes of IBs of two adjacent energy bands may differ less than $0.1^{\circ}$. However, uncertainty in calibration factors and other reasons (finite temporal resolution, temporal evolution during auroral oval crossings, etc.) only allow the determination of the IB location inside some "confidence interval". We define the boundaries of this interval as follows (an example is given assuming that the satellite moves from the equator to the pole): the equatorial boundary is the polewardmost point where $F^{0} / F^{90}<0.5$ and $F^{0} / F^{90}<0.5$ for the 4 preceding points (8-s interval); the polar boundary is the first point after the equatorial boundary where $F^{0} / F^{90}>0.75$ and $F^{0} / F^{90}>0.75$ for 4 subsequent points. Further in the text, the equatorial and polar latitudes of the IB confidence interval will be referred to as $\Lambda_{\mathrm{eq}}$ and $\Lambda_{\mathrm{pol}}$, respectively. The criterion for $\Lambda_{\text {eq }}$ was chosen so that it ignores brief periods of isotropic or nearly isotropic fluxes at the equatorial part of auroral oval which probably are caused by a wave-particle interaction scattering mechanism (Gvozdevsky et al., 1997; Yahnin and Yahnina, 2007). These criteria were used for determination of the confidence interval for P1 and P2 IBs. The P3 IB was determined using similar criteria with the weaker condition on the number of points preceding $\Lambda_{\text {eq }}$ and following $\Lambda_{\text {pol }}$ ( 2 instead of 4$)$. The absolute values of latitudes are used for observations in the Southern Hemisphere in order to combine the observations from both hemispheres.

We applied this algorithm to the auroral oval crossings in both hemispheres in 17:00-24:00, 00:00-07:00 MLT sector in 00:00-12:00 UT interval. After that, we visually inspected all IBs and excluded an insignificant number of incorrect ones. The events with large uncertainty usually correspond to a situation when the regions of isotropic and strongly anisotropic fluxes were separated by a region of transient and weak deviations from isotropy so that the fluxes cannot be considered as purely isotropic or strongly anisotropic. Finally, we have a database of $\sim 250$ proton IB positions for P1, P2, P3 energy bands with information about their uncertainty. On average, the measured counts are one order of magnitude lower for each following energy band. For that reason count statistics for P3 band is poorest, with MEPED P3 $0^{\circ}$ counts being less than 10 for some of orbits (basically during the prestorm interval). We discarded the IB if the $0^{\circ}$ flux counts were less than 10 . It led to a significantly smaller number of P3 IBs detected in comparison with P1 and P2 IBs.

\subsection{IB energy dispersion analysis}

In further analysis we distinguished three IB dispersion types that are illustrated in Fig. 4. This schematic figure shows latitude profiles of particle fluxes for $0^{\circ}$ (black curve) and $90^{\circ}$ (red curve) detectors for P1 and P2 bands. The IB confidence intervals are marked by vertical lines. Figure $4 \mathrm{a}$ illustrates the energy dispersion expected for scattering on curved field lines (the P2 IB confidence interval is situated equatorward of that for $\left.\mathrm{P} 1, \mathrm{P} 1 \Lambda_{\mathrm{eq}}>\mathrm{P} 2 \Lambda_{\mathrm{pol}}\right)$. This type is referred to as "ND" (normal dispersion). Figure $4 \mathrm{~b}$ illustrates the opposite situation $\left(\mathrm{P} 2 \Lambda_{\mathrm{eq}}>\mathrm{P} 1 \Lambda_{\mathrm{pol}}\right)$. We cannot relate this type of dispersion with any certain isotropisation mechanism and we will refer to this type as "AD" (anomalous dispersion). Figure $4 \mathrm{c}$ and d illustrate the situation when IB confidence intervals of two energy bands overlap and we can not relate this event with any of the aforementioned types. This dispersion type is referred to as "UD" (unidentified dispersion). These definitions also can be generalized to the usage of three energy bands P1, P2, P3: If every pair of bands has ND dispersion type, we define this event as ND type. If at least one of the pairs is of $\mathrm{AD}$ dispersion type, this event is referred to as AD type. All remaining events are classified as UD type.

Figure 5a, b, c show locations of observed P2 IB as the corrected geomagnetic latitude (CGMLat) vs. MLT for 00:00 12:00 UT interval. Red, black and blue symbols correspond to ND, AD and UD dispersion types respectively. The differences of the three figures are due to the different combination of energy bands used for classification of the IB dispersion types. All three energy bands were used for dispersion classification presented in Fig. 5a, whereas Fig. 5b and c are obtained using P1, P2 and P2, P3 pairs, respectively. There are fewer points in Fig. $5 \mathrm{a}$ and $\mathrm{c}$ in comparison with Fig. $5 \mathrm{~b}$ because $\mathrm{P} 30^{\circ}$ flux often does not rise above the 10 -count limit and P3 IB cannot be reliably determined. It is obvious that that P3, P2 pair generally exhibits ND dispersion type (no AD type points in Fig. 5c). This important finding may mean that the physical mechanism leading to anomalous dispersion only affect the particles in the lowest energy range ( 30-80 keV); however, other explanations are also possible. Keeping this fact in mind, we will focus on the analysis of dispersions of the P1 and P2 IBs. Figure 5a and b look similar except for a larger number of points in the latter. Both figures show that AD dispersion types tend to be observed in the dusk-midnight MLT sector with only one point in the morning sector. However, one should keep in mind the large number of UD type points in the morning sector. Since most of AD type points in Fig. 5a are also present in Fig. 5b, for the sake of better statistics we further will determine the dispersion type using only the P1, P2 IB pair.

To study the occurrence rate of the different types of the IB energy dispersion during a particular storm phase, we selected a few specific periods presented in Table 2. The first period is a "prestorm" period, when Sym-H* is more or less stable (does not show a steep decrease). The names "main1" 

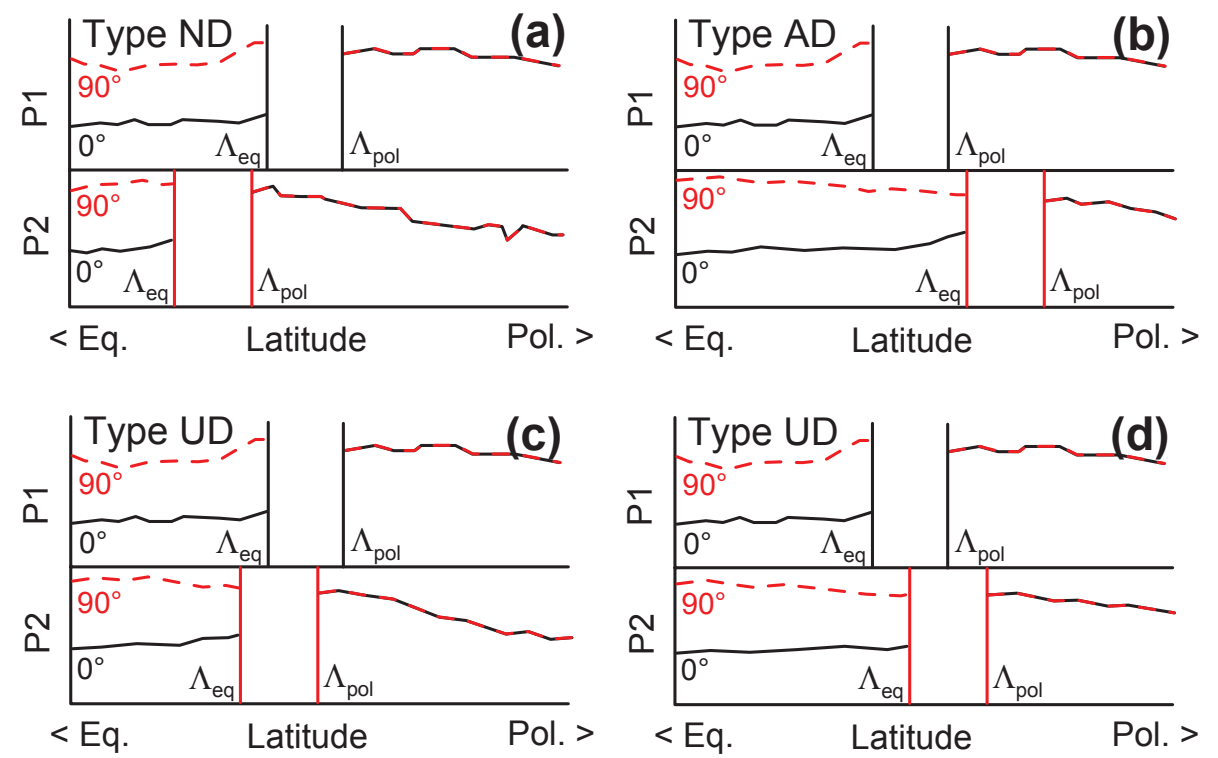

Fig. 4. Sketches illustrating criteria for definition of three IB energy dispersion types. P1 and P2 panels correspond to lower and higher energy bands. Vertical lines mark confidence intervals of IB determination ( $0^{\circ}$ and $90^{\circ}$ fluxes are not shown inside these intervals).

Table 2. Statistics of observation of different types of IB energy dispersion during specific phases of the storm. The phases are defined according to Sym- $\mathrm{H}^{*}$. IB dispersion type was determined for P1, P2 energy bands.

\begin{tabular}{lcccccrrr}
\hline Phase & Interval UT & $\mathrm{N}$ & $\mathrm{ND}$ & $\mathrm{AD}$ & $\mathrm{UD}$ & $\mathrm{ND} / \mathrm{N}$ & $\mathrm{AD} / \mathrm{N}$ & $\mathrm{UD} / \mathrm{N}$ \\
\hline prestorm & $00: 00-02: 00$ & 13 & 6 & 1 & 6 & $46 \%$ & $8 \%$ & $46 \%$ \\
main1 & $02: 00-05: 20$ & 20 & 1 & 3 & 16 & $5 \%$ & $15 \%$ & $80 \%$ \\
recov1 & $05: 20-08: 30$ & 23 & 6 & 2 & 15 & $26 \%$ & $9 \%$ & $65 \%$ \\
main2 & $08: 30-09: 10$ & 5 & 0 & 0 & 5 & $0 \%$ & $0 \%$ & $100 \%$ \\
recov2 & $09: 10-12: 00$ & 13 & 9 & 0 & 4 & $69 \%$ & $0 \%$ & $31 \%$ \\
\hline
\end{tabular}

and "main2" denote the periods of sharp Sym- $\mathrm{H}^{*}$ decrease. "Recov1" is the period of temporary Sym- $\mathrm{H}^{*}$ recovery between the two dips and "recov2" is the first part of the main storm recovery period. Columns 4-6 of Table 2 summarize the number of points of the specific dispersion type during the given period and the third column is the total number of points. Columns 7-9 show the percentage of the points of that specific type. Figure 6a graphically presents the data of columns 7-9 of Table 2. It can be seen that the occurrences of ND and UD dispersion types (red and blue bars) behave in the opposite way. The ND occurrence rate is higher during quiet intervals (prestorm and recovery periods) whereas the UD occurrence is higher during both $\mathrm{Sym}-\mathrm{H}^{*}$ periods. This behavior can be interpreted as a manifestation of the strengthening of the radial gradient of the magnetic field in the inner magnetosphere during the main phase so that the P1 and P2 IBs become closer and our algorithm often can not resolve them (the number of UD dispersions increases). The occurrence of AD type peaks during the first Sym- $\mathrm{H}^{*}$ dip and it is also observed during the prestorm and first Sym- $\mathrm{H}^{*}$ recovery periods. It should be noted that activity in the auroral region did not cease immediately after the Sym- $\mathrm{H}^{*}$ minimum. Although the AL index recovers gradually (see Fig. 1c), this recovery is interrupted by brief intensifications until 07:10 UT. For that reason we tried another classification of activity periods based on the level of AL index disturbance/variability. The periods are specified in Table 3 . The names "dist 1 " and "dist2" represent the periods of disturbed AL index, whereas the "quiet" periods correspond to the quiet AL. The occurrence rates of different dispersion types are shown in Table 3 and in Fig. 6b in the same format as in Table 2 and Fig. $6 \mathrm{a}$. The AL-based classification seems to give a more consistent picture. The occurrences of ND and UD dispersions behave in the same way as in Fig. 6a. The ND occurrence rate is $\sim 50 \%$ during the prestorm and quiet periods and it is less than $20 \%$ during disturbed periods. Almost all anomalous dispersions were observed during the first disturbed period ("dist1"). The only exception was observed during the prestorm interval (see Table 3). If the AD dispersion type is somehow related to substorm injections, one would expect $\mathrm{AD}$ dispersion observed during the second $\mathrm{AL}$ intensification too. However, this second intensification period was too 

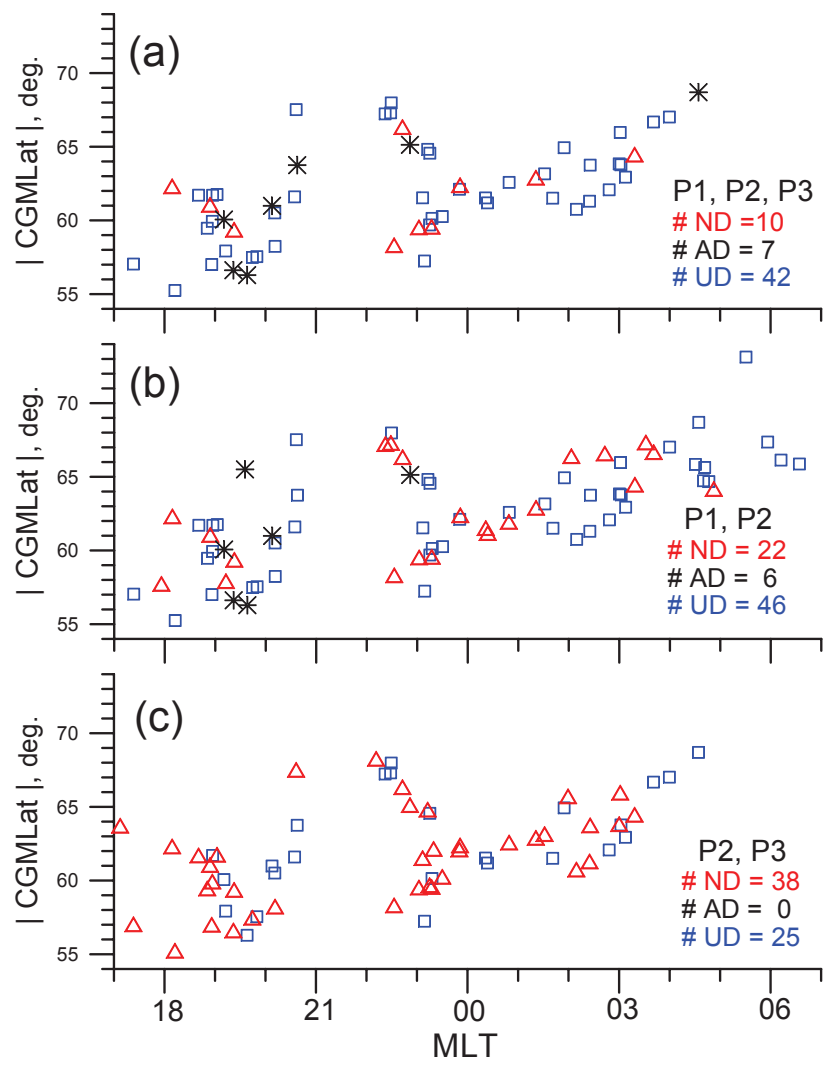

Fig. 5. CGMLat vs. MLT plot of P2 IB positions. Red triangles correspond to ND type, black asterisks to $\mathrm{AD}$ type and blue squares to UD type. Panels correspond to different dispersion type classification methods. (a) involving P1, P2, P3 channels, (b) P1 and P2 channels, (c) P1 and P3 channels, see explanation in the text.

short and only seven IBs were observed during this period. Taking into account that $\mathrm{AD}$ occurrence rate never exceeded $14-15 \%$, this type of dispersion could be easily missed by a satellite during the "dist2" interval. The occurrence rates in Tables 2 and 3 were computed relative to the number of all events when P1 P2 IBs were determined including UD type events. If we compute the $\mathrm{AD}$ occurrence rate relative to the number of events when AD or ND dispersion type was identified, $75 \%$ and $56 \%$ rates will be found for "main1" and "dist 1 " periods, respectively. However, taking into account the limited number of events for analysis and large number of UD type points, these occurrence rates should not be used for comparison with other studies.

\subsection{Comparison with the model IBs}

The analysis of the measurements in the vicinity of geostationary orbit (Sect. 3) has shown that a relatively thin and intense current sheet exists in the dusk sector and the TS05 model underestimates magnetic field line stretching in this region. On the other hand, the analysis of the IB energy dispersion (Sect. 5.3) has shown that the anomalous IB disper-
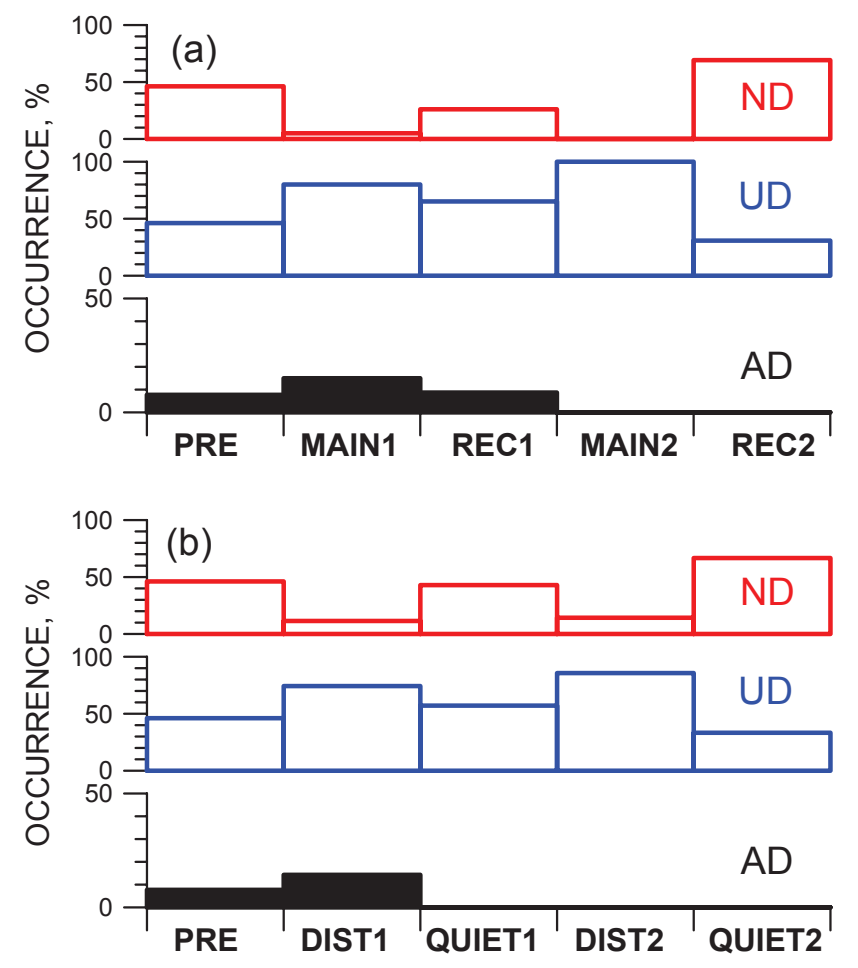

Fig. 6. Occurrence rate of different types of IB dispersions for specific phases of the storm: (a) phases defined according to Sym- $\mathrm{H}^{*}$, (b) phases defined according to the AL index.

sion type is mostly observed in the dusk and premidnight MLT sectors. However, it was also shown that P2 and P3 IBs never exhibit anomalous dispersion (Fig. 5c). Although it does not necessarily mean that P2 and P3 isotropic boundaries are formed by scattering on curved field lines in the current sheet region, we compare the observed P2 IBs to the model ones.

Locations of P2 IBs in the Northern Hemisphere for different MLT were computed using the criterion $R_{\mathrm{c}} / \rho=8$ (see Sect. 5.1) for the TS05 magnetic configuration and for a particle energy of $80 \mathrm{keV}$. The computations were performed for the period of 00:00-12:00 UT with 1-h resolution for corresponding input parameters. Figure 7 shows the corrected geomagnetic latitude and MLT of the observed (symbols) and model (curves) IBs. Colors represent the corresponding Sym- $\mathrm{H}^{*}$ index value. An absolute value of latitude is plotted for observations in the Southern Hemisphere. Figure 7a, $\mathrm{b}$, c, shows IB positions during the prestorm interval, disturbed period, and the recovery period, respectively. The exact time intervals are specified in the figure. The high occurrence of ND dispersion type during the prestorm and recovery periods (see Tables 2 and 3) allows us to interpret the IB position in Fig. 7a, $\mathrm{c}$ in terms of nightside cross-tail current strength as it was discussed in Sect. 5.1. Figure 7a demonstrates that TS05 can overestimate cross-tail current strength during the prestorm period, especially in the dusk 
Table 3. The same as Table 2 but storm phases are defined according to the AL index.

\begin{tabular}{lccccccrc}
\hline Phase & Interval UT & $\mathrm{N}$ & $\mathrm{ND}$ & $\mathrm{AD}$ & $\mathrm{UD}$ & $\mathrm{ND} / \mathrm{N}$ & $\mathrm{AD} / \mathrm{N}$ & $\mathrm{UD} / \mathrm{N}$ \\
\hline prestorm & $00: 00-02: 10$ & 13 & 6 & 1 & 6 & $46 \%$ & $8 \%$ & $46 \%$ \\
dist1 & $02: 10-07: 10$ & 35 & 4 & 5 & 26 & $11 \%$ & $14 \%$ & $74 \%$ \\
quiet1 & $07: 10-08: 10$ & 7 & 3 & 0 & 4 & $43 \%$ & $0 \%$ & $57 \%$ \\
dist2 & $08: 10-09: 30$ & 7 & 1 & 0 & 6 & $14 \%$ & $0 \%$ & $86 \%$ \\
quiet2 & $09: 30-12: 00$ & 12 & 8 & 0 & 4 & $67 \%$ & $0 \%$ & $33 \%$ \\
\hline
\end{tabular}

and premidnight sector. It is also the case for the dawnside during the recovery period (Fig. 7c). Note, however, that the points in the dusk sector are in good agreement with TS05 during the recovery phase.

The storm has two intensifications separated by the period of temporary Sym-H* recovery (Fig. 1). For simplicity, we do not separate these periods combining all the data during the 02:10-09:30 interval in Fig. 7b. However, the comparison of observed P2 IBs to the model ones in Fig. 7b should be made with precautions. The P2, P3 pair of IBs never shows anomalous dispersion, while the $\mathrm{P} 1, \mathrm{P} 2$ pair sometimes does. At least two hypotheses can be considered: (1) The anomalous scattering mechanism only affects the $\mathrm{P} 1$ energy range particles, whereas criterion $R_{\mathrm{c}} / \rho=8$ can be used for $\mathrm{P} 2$ and P3 IBs. (2) The anomalous scattering mechanism affects the particles in all energy ranges, but the normal dispersion of P2 and P3 IBs is caused by some other reason. Of course, the interpretation of the results presented in Fig. 7 depends on the choice of hypothesis. In general, Fig. 7b demonstrates that the TS05 model underestimates the dusk-dawn asymmetry of the equatorial current during the active phase of the storm because. According to Fig. 5b, there are two ND type points below $58^{\circ}$ CGMLat in the dusk sector and there are no AD type points in the midnight-dawn sector at all. The observed IBs in the duskside sector are on average shifted $\sim 3-5^{\circ}$ equatorward relative to IBs in the midnight sector. There is a group of IBs in the 17:00-20:00 MLT sector which are $3-4^{\circ}$ equatorward of the model IB. The two most equatorial IBs (lat.: 55-56.5 ${ }^{\circ}$ ) were observed during the main Sym-H* minimum (red color) and were of AD and UD types (see Fig. 5). Three of the most polar IBs around $\sim$ 19:00 MLT were observed during the temporary recovery (06:45-08:30 UT).

To check how the PRC intensity influences the IB location, we computed IB positions using TS05 with an increased PRC intensity by a factor $\times 2, \times 5$ and $\times 10$. We found that only the factor $\times 5$ can produce an IB at $\sim 57^{\circ}$ magnetic latitude on the duskside for this event and a factor $\times 10$ produces an IB at $\sim 55^{\circ}$ (the lowest latitude of observed IB).

It should be noted that times of strongest $B_{\mathrm{r}}$ on the duskside (Fig. 3a, c, d) coincide (within $15 \mathrm{~min}$ ) with times of the two Sym- $\mathrm{H}^{*}$ minima. To look at this tendency from a different angle, we analyze isotropic boundary evolution in the dusk sector. Figure 8 shows the stacked latitudinal profiles

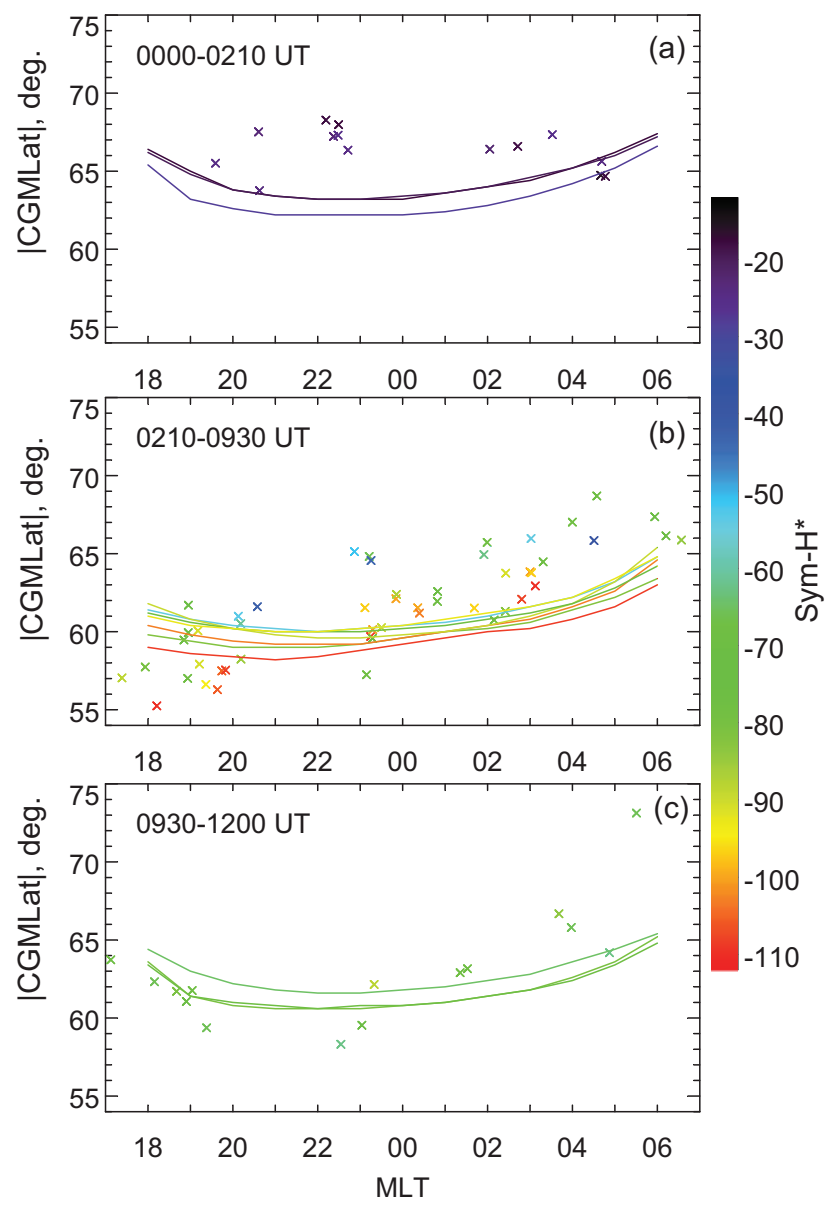

Fig. 7. Observed (symbols) and model (curves) isotropic boundaries during (a) prestorm interval, (b) active phase of the storm and (c) recovery phase, shown as CGMLat vs. MLT. Color shows corresponding Sym- $\mathrm{H}^{*}$ index values.

of $0^{\circ}$ and $90^{\circ} \mathrm{P} 2$ flux. All profiles correspond to observations in the 17-20.5 MLT sector and plotted chronologically from the top to the bottom. The Universal Time is shown on the left, and the values of Sym- $\mathrm{H}^{*}$ are shown on the right. The horizontal axis represents an absolute value of corrected geomagnetic latitude. Spacecraft IDs are shown as vertical axis titles. NOAA-15 and -16 passed the given MLT sector in the Southern Hemisphere, whereas METOP and NOAA17 (marked by red color) did in the Northern Hemisphere. 


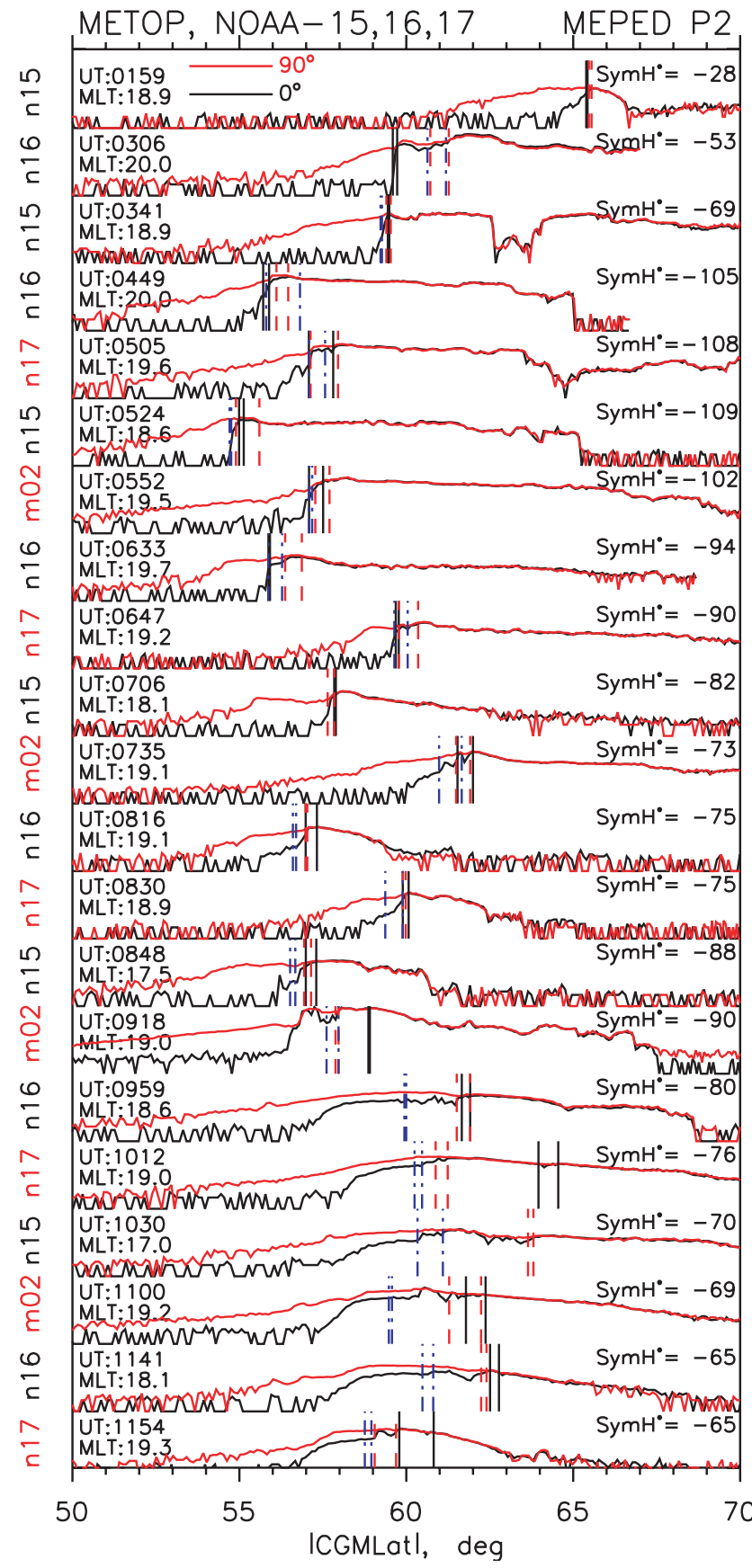

Fig. 8. Latitudinal profiles of proton number flux (MEPED P2, in $\log$ scale) in 17:00-20:00 MLT sector. The panels are plotted chronologically. The MLT and UT on right and Sym-H* on the left correspond to the time the satellite crossed of $60^{\circ}$ CGMLat. Satellite name is shown at the vertical axis and the red (black) colors correspond to the auroral oval crossings in the Northern (Southern) Hemispheres. Vertical lines mark IB positions (P1 black; P2 red and P3 blue).

The vertical black, red, and blue lines mark the IB confidence intervals for P1, P2, P3 energy bands, respectively.
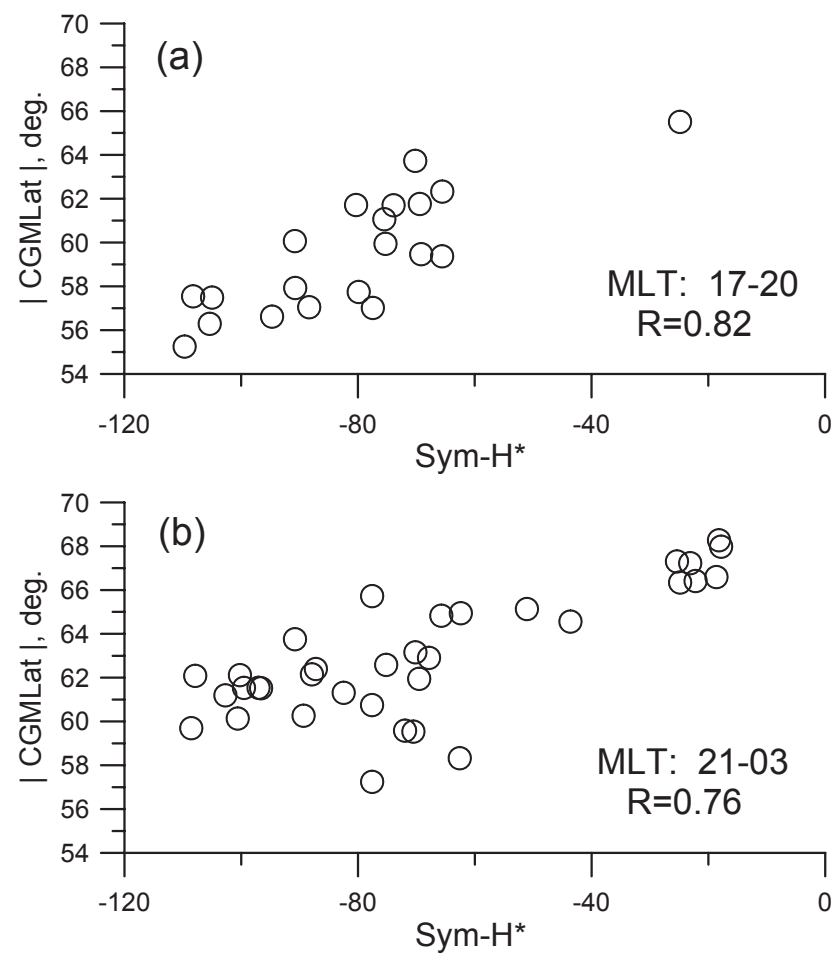

Fig. 9. P2 IB latitude vs. Sym-H* in the 17:00-20:00 MLT sector (a) and in the 21:00-03:00 MLT sector (b).

A general tendency is that IBs move to the equator when Sym- $\mathrm{H}^{*}$ decreases, and retreat back to the pole when it recovers. It is true for all energy bands because the Sym- $\mathrm{H}^{*}$ dependent variation of IB positions is much stronger than IBs energy dispersion. It was shown in Sect. 5.3 and it is also seen from Fig. 8 that P3 and P2 IBs (blue and red vertical lines) mostly exhibit ND dispersion type. Figure 9 shows P2 IB CGMLat vs. Sym-H*. Figure 9a corresponds to the IBs in the dusk sector 17:00-20:00 MLT, whereas Fig. 9b shows those determined in the 21:00-03:00 MLT sector. The linear correlation coefficient between the IB latitude and Sym- $\mathrm{H}^{*}$ were computed. We found a strong correlation in the dusk sector with $r=0.82(N=20)$ and somewhat weaker correlation in the midnight sector $(r=0.76, N=34)$. The correlation coefficient between the IB latitude and the AL index were much weaker at 0.56 and 0.13 , respectively.

\section{Discussion}

Although we cannot determine which isotropization mechanism operated in our case, the spatial and temporal relation between current sheet thinning and the equatorward IB shift is evidence in favor of scattering on curved field lines. The TS05 model underestimates the $B_{\mathrm{r}}$ on the duskside severely, hence the real IBs should lie equatorward of the model curves in Fig. 7. Tsyganenko and Sitnov (2005) found that a thin current sheet can approach very near to Earth on the nightside 
as close as the geocentric distance $\sim 3-4 R_{\mathrm{E}}$ during the peak of the superstorm. So why can it not approach the same distance on the duskside?

However, caution should be used when applying $R_{\mathrm{c}} / \rho=8$ criterion for interpretation of IB observations during disturbed periods. Søraas et al. (2002) interpreted the equatorial part of the energetic proton precipitation in the evening sector as a freshly injected isotropic plasma. There are many observations supporting a wave-scattering scenario. Mostly, these are magnetospheric and low-altitude observations of the EMIC wave activity in the inner magnetosphere region (Bräysy et al., 1998; Erlandson and Ukhorskiy, 2001; Halford et al., 2010). Gvozdevsky et al. (1997) investigated the intense proton precipitation equatorward of the IB which were called LLPP (low-latitude proton precipitation). The examples of such precipitation can be seen in Fig. 8 (last six profiles). The authors found that LLPP particle flux significantly increases during intense substorms, but also mentioned that sometimes they could not recognize LLPP during a substorm maximum epoch. They discussed two possible reasons: first, the equatorward motion of IB during disturbed time (the isotopic zone can completely overlap the LLPP region). Second, the increase of the pitch angle diffusion rate so that the fluxes become fully isotropic and the LLPP region cannot be distinguished from isotropic precipitation caused by scattering on the curved field lines. In the latter case, the isotropic boundary is formed by a wave-scattering mechanism and the criterion $R_{\mathrm{c}} / \rho=8$ cannot be used.

The MLT distribution of anomalous IB dispersion (Fig. 5b) resembles the distribution of EMIC waves observed by Erlandson and Ukhorskiy (2001). However, the authors found higher occurrence during the recovery phase than during the main phase. Halford et al. (2010) indeed found that the majority of EMIC waves occur during the main phase but most of the events were observed in the dusk-noon sector and relatively few were observed for MLT $>18 \mathrm{~h}$, whereas there were no anomalous IB dispersions observed for MLT $<19 \mathrm{~h}$. It is also unclear why higher energy IBs never exhibit anomalous dispersion (Fig. 5c). The decrease of equatorial magnetic field in the inner magnetosphere due to the duskside current strengthening can create favorable conditions for EMIC waves generation and ion pitch angle diffusion (Kennel and Petschek, 1966). In this case, again, the IB latitude can be considered as an indicator of equatorial current strength.

However, we have presented an analysis of one event and the question arises as to how typical the event might be. Inspection of previous statistical results show that all these findings are inherent features of a magnetic storm.

A manifestation of a strong current on the duskside can be seen in the GOES statistical observations during the main and recovery phases (Ohtani et al., 2007). Comparison of their Fig. $2 b$ and $f$, showing the disturbance of the radial component of the magnetic field at 03:00-06:00 and 18:0021:00 MLT sectors, demonstrates that the dawn-dusk asym- metry increases with a Sym-H decrease in agreement with our results. The asymmetry is also seen in the depression of $B_{\mathrm{Z}}$ (their Fig. 4a, b).

Statistical comparisons of observed $B_{\mathrm{x}}, B_{\mathrm{y}}, B_{\mathrm{z}}$ GSM components at geosynchronous orbit during storm times and their values predicted by the TS05 model revealed the worst correlation for $B_{\mathrm{y}}$ (Tsyganenko and Sitnov, 2005). Their scatter plots show that the $B_{\mathrm{y}}$ difference can be $>100 \mathrm{nT}$ (Tsyganenko et al., 2003). However, the y-axis is close to the radial direction if the spacecraft is on the dusk or dawn flanks and strengthening of the duskside current sheet might be responsible for this discrepancy.

Our findings are in agreement with results of a storm empirical model by Tsyganenko and Sitnov (2007) (by now, the model is available for the list of processed storms, which does not include our studied event). This model does not include predefined current systems, but rather expands the magnetic field into a sum of specific basis functions with coefficients which are found by fitting to the data. Even though the function defining the current sheet thickness variation over the equatorial plane is defined a priori and is an even function of $Y$, the model shows that there is a strong current on the duskside during the main phase. Unlike the conventional partial ring current, the model current sheet extends from 5 to $>10 R_{\mathrm{E}}$ in radial distance and closes basically on the magnetopause.

The determined latitudes of IBs in the dusk sector during the Sym- $\mathrm{H}^{*}$ minimum period are in agreement with the statistical study of the proton isotropy boundary by Lvova et al. (2005). The authors studied the latitude MLT shape of the IB as a function of solar wind parameters and geomagnetic indices. It was found that the IB can reach $\sim 54-55^{\circ}$ during disturbed times (Dst $<-100 \mathrm{nT}$ ) in the premidnight sector.

Keeping in mind that the latitude of the IB is a good indicator of the equatorial current strength, our results on the MLT dependence of the IB latitude are in agreement with the study of Le et al. (2004), who determined the 3-dimensional current density by taking the curl of the statistically determined magnetic field. The authors found that the total current (integrated from 4 to $8 R_{\mathrm{E}}$ in $r$ and -2 to $2 R_{\mathrm{E}}$ in $Z$ ) has a maximum in the 19:00-21:00 MLT sector for Sym-H $<-60 \mathrm{nT}$. However, the Le et al. (2004) dataset includes both the main and recovery phases, hence the main phase asymmetry can be even stronger.

While none of these previous studies explicitly proves the existence of the thin and strong duskside current sheet, as a group they lend credibility to the concept that such a current could exist, at least temporarily, during the main phase of the storm.

The IBs in the dusk sector were on average shifted $\sim 3-$ $5^{\circ}$ equatorward relative to IBs in the midnight sector. This allows us to suggest that the duskside current can be even stronger and flows closer to Earth than the tail current in the midnight sector. The correlation of the IB latitude with Sym$\mathrm{H}^{*}$ in the dusk sector was also somewhat better than that in 
the midnight sector (Fig. 9). It might also mean that the duskside current contributes more to Sym-H than the tail current in the midnight sector; however, this suggestion requires a quantitative evaluation.

\section{Conclusions}

Our analysis of a moderate storm on 22 July 2009 revealed the following:

1. A very strong radial component of the magnetic field was observed in the 18:00-20:00 MLT sector in the vicinity of geosynchronous orbit indicating the development of a highly stretched configuration with $\left|B_{\mathrm{r}}\right|>>$ $\left|B_{\mathrm{Z}}\right|$ during the main phase.

2. A rough estimation of the current sheet half-thickness in the dusk sector gives the value $\leq 1 R_{\mathrm{E}}$.

3. Our tests show that TS05 fails to reproduce the duskside magnetic field during this particular storm. Moreover, neither a change of azimuthal angle of the PRC maximum nor a variation of its intensity can describe the observed large $B_{\mathrm{r}}$ in the dusk sector.

4. Analyzing the dispersion of proton isotropic boundaries (IBs) we found that IBs of the high-energy pair of channels $(E \gtrsim 100 \mathrm{keV}$ ) always exhibit normal dispersion (dispersion type expected for pitch angle scattering on curved field lines; IB of the high-energy particles is observed at lower latitude than IB of the lower energy particles). However, we found six anomalous IB dispersion events (of 28 when dispersion type identification could be done) for the two low-energy channels $(E \lesssim 100 \mathrm{keV})$.

5. All anomalous dispersion events were observed in the dusk-midnight MLT sector and five of them were observed during the first AL index intensification. The occurrence of anomalous dispersion is not directly related to storm phase. One event was observed during the prestorm interval and two other events were observed during first Sym- $\mathrm{H}^{*}$ recovery period of 06:3006:50 UT.

6. Analyzing the $\sim 80 \mathrm{keV}$ proton IB location during the storm peak period we found that the observed IBs in the 17:00-20:00 MLT sector reached $\sim 55^{\circ}$ magnetic latitude. This is $\sim 4^{\circ}$ equatorward of the model IB. The duskside IBs were on average shifted $\sim 3-5^{\circ}$ equatorward relative to IBs in the midnight sector.

7. The latitude of IBs in the 17:00-20:00 MLT sector correlates with the Sym-H* index with $r=0.82$ and the correlation is somewhat better than in the midnight sector where it might be influenced by substorms.
8. The difference of $4^{\circ}$ between the observed IBs and the TS05 model IBs in the dusk sector can be achieved by introducing an unrealistic $\times 10$ factor to the model PRC intensity.

All of the above findings support a hypothesis that a relatively thin (half-thickness $<1 R_{\mathrm{E}}$ ) sheet of current flowing in the azimuthal direction developed on the duskside during the storm main phase. Its sheet-like shape distinguishes this current from the conventional PRC, which has a bean-shaped cross section. We cannot determine how this current closes and whether it is linked to energetic particle injections; however, results of the Tsyganenko and Sitnov (2007) modeling support the hypothesis that its topology is closer to the tail current flowing out through the magnetopause.

\section{Appendix A}

\section{Calibration of MEPED $90^{\circ}$-flux data}

According to Asikainen et al. (2012) the impact of radiation damage on the MEPED detectors leads to a shift of the energy thresholds to higher values. Unfortunately, the $90^{\circ}$ detector degrades faster than $0^{\circ}$ detector and reliable IB identification becomes impossible after 1-2 yr of satellite operation. The accuracy of IB determination depends only on relative calibration of $0^{\circ}$ and $90^{\circ}$ detectors. On the other hand, it has been known since the first low-altitude particle observations (Søraas et al., 1977) that there is a region of fully isotropic flux at high latitudes. A calibration of $90^{\circ}$ detectors with respect to $0^{\circ}$ detectors can be done using the measurements in this isotropy region. We used the following criteria to select the isotropy region during every auroral oval crossing during 22 July 2009: (1) Only measurements poleward of $0^{\circ}$-flux maximum were used; (2) the measured $0^{\circ}$ flux is higher than the $90^{\circ}$ flux; (3) MLT $>17 \mathrm{~h}$ or MLT $<7 \mathrm{~h}$; (4) we use a 10-count lower limit for the P1 and P2 bands and a 5-count lower limit for the P3 band. The median of $0^{\circ} / 90^{\circ}$-flux ratio (with standard deviation) in the isotropy region is summarized in Table A1 for METOP, NOAA-15, -16, $-17,-18$ satellites (the NOAA-19 had been recently launched and its data did not need a calibration). These ratios can be used as correction factors for $90^{\circ}$-flux measurements, however, standard deviations are rather large. If the difference of the measured $0^{\circ}$ and $90^{\circ}$ fluxes in the isotropy region is a result of the different low-energy thresholds, this ratio depends on the slope of the particle energy spectrum. The spectrum slope is roughly proportional to the ratio of counts in two adjacent energy bands. Our idea was to find the empirical dependence between the calibrating factor $\left(0^{\circ} / 90^{\circ}\right.$-flux ratio in the isotropy region) and the ratio of the fluxes in two adjacent energy bands and to use this dependence to find a calibrating factor for every measurement. We use the P2/P1 $90^{\circ}$-flux ratio as a measure of the spectrum slope for calibration of $\mathrm{P} 1$ and $\mathrm{P} 2$ fluxes and $\mathrm{P} 3 / \mathrm{P} 290^{\circ}$-flux ratio as a measure of the 
Table A1. ( $0^{\circ} / 90^{\circ}$-flux ratio)/(standard deviation) for $\mathrm{P} 1, \mathrm{P} 2, \mathrm{P} 3$ proton energy bands.

\begin{tabular}{lccc}
\hline Sat. name & P1 & P2 & P3 \\
\hline METOP & $1.17 / 0.20$ & $1.35 / 0.46$ & $1.38 / 0.39$ \\
NOAA-15 & $1.88 / 0.40$ & $2.13 / 0.51$ & $1.95 / 0.75$ \\
NOAA-16 & $1.68 / 0.71$ & $2.33 / 1.27$ & $1.54 / 0.46$ \\
NOAA-17 & $1.51 / 0.30$ & $1.86 / 0.56$ & $1.89 / 0.48$ \\
NOAA-18 & $1.14 / 0.15$ & $1.24 / 0.28$ & $1.20 / 0.33$ \\
\hline
\end{tabular}

Table A2. Linear correlation coefficient/number of data points. The correlation coefficient between $0^{\circ} / 90^{\circ}$-flux ratio in the isotropic region and the parameter representing the energy spectrum slope for corresponding energy band (see explanation in the text).

\begin{tabular}{lccc}
\hline Sat. name & $\mathrm{P} 1$ & $\mathrm{P} 2$ & $\mathrm{P} 3$ \\
\hline METOP & $-0.31 / 2097$ & $-0.53 / 2175$ & $-0.41 / 921$ \\
NOAA-15 & $-0.69 / 1147$ & $-0.06 / 951$ & $-0.58 / 149$ \\
NOAA-16 & $-0.57 / 1359$ & $-0.63 / 1435$ & $-0.32 / 175$ \\
NOAA-17 & $-0.53 / 1883$ & $-0.46 / 1776$ & $-0.36 / 472$ \\
NOAA-18 & $-0.25 / 1723$ & $-0.51 / 1465$ & $-0.30 / 353$ \\
\hline
\end{tabular}

slope for calibration of P3 flux. The correlation coefficients ( $R$, computed for logarithm values) are summarized in Table A2 with the number of data points.

We performed a linear fit of the logarithm values for all satellites/energy bands except for NOAA-15 P2 (which shows no correlation) and NOAA-16 P3 (there were few data points and the dependence looked strange). For these two we use the median of the calibrating factors from Table A1. To minimize the influence of outliers on the resulting fit, we smoothed the original scattered dependence using the median filter. The 50 data point filtering window size was chosen for the P1 and P2 energy bands and 10 data point size for the P3. These smoothed dependences were fitted by a linear equation using an ordinary least-square method. These linear regression coefficients were used to find a calibration factor for every measurement of $90^{\circ}$ flux.

Acknowledgements. The authors thank N. A. Tsyganenko for his help with the TS05 code. Work by SD was supported by an Academy of Finland grant. Magnetospheric indices and solar wind parameters were obtained from the OMNI database provided by J. H. King, N. Papitashvilli at AdnetSystems, NASA GSFC. GOES data were obtained via the CDAWeb. NOAA MEPED particle data have been made publicly available by NOAA. Thanks to K. H. Glassmeier, U. Auster, and W. Baumjohann for the use of FGM data provided under the lead of the Technical University of Braunschweig and with financial support through the German Ministry for Economy and Technology and the German Center for Aviation and Space (DLR) under contract 50 OC 0302. The authors thank the International Space Science Institute in Bern, Switzerland, for their support of an international team on "Resolving Current Systems in Geospace". Work by NG and ML was supported by
NASA and NSF grants.

Topical Editor I. A. Daglis thanks N. A. Tsyganenko and one anonymous referee for their help in evaluating this paper.

\section{References}

Asikainen, T., Maliniemi, V., and Mursula, K.: Modeling the contributions of ring, tail, and magnetopause currents to the corrected Dst index, J. Geophys. Res., 115, A12203, doi:10.1029/2010JA015774, 2010.

Asikainen, T., Mursula, K., and Maliniemi, V.: Correction of detector noise and recalibration of NOAA/MEPED energetic proton fluxes, J. Geophys. Res., 117, A09204, doi:10.1029/2012JA017593, 2012.

Bräysy, T., Mursula, K., and Marklund, G.: Ion cyclotron waves during a great magnetic storm observed by Freja double-probe electric field instrument, J. Geophys. Res., 103, 4145-4155, doi:10.1029/97JA02820, 1998.

Cahill Jr., L. J.: Inflation of the Inner Magnetosphere during a Magnetic Storm, J. Geophys. Res., 71, 4505-4519, doi:10.1029/JZ071i019p04505, 1966.

Crooker, N. U. and Siscoe, G. L.: Birkeland Currents as the Cause of the Low-Latitude Asymmetric Disturbance Field, J. Geophys. Res., 86, 11201-11210, doi:10.1029/JA086iA13p11201, 1981.

Cummings, W. D.: Asymmetric Ring Currents and the LowLatitude Disturbance Daily Variation, J. Geophys. Res., 71, 4495-4503, doi:10.1029/JZ071i019p04495, 1966.

Erlandson, R. E. and Ukhorskiy, A. J.: Observations of electromagnetic ion cyclotron waves during geomagnetic storms: Wave occurrence and pitch angle scattering, J. Geophys. Res., 106, 3883 3895, doi:10.1029/2000JA000083, 2001.

Evans, D. S. and Greer, M. S.: Polar orbiting environmental satellite space environment monitor: 2 . Instrument descriptions and archive data documentation, Tech. Memo., OAR SEC-93, NOAA, Boulder, Colorado, 2000.

Ganushkina, N. Y., Dubyagin, S., Kubyshkina, M., Liemohn, M., and Runov, A.: Inner magnetosphere currents during the CIR/HSS storm on July 21-23, 2009, J. Geophys. Res., 117, A00L04, doi:10.1029/2011JA017393, 2012.

Gvozdevsky, B. B., Sergeev, V. A., and Mursula, K.: Long lasting energetic proton precipitation in the inner magnetosphere after substorms, J. Geophys. Res., 102, 24333-24338, doi:10.1029/97JA02062, 1997.

Halford, A. J., Fraser, B. J., and Morley, S. K.: EMIC wave activity during geomagnetic storm and nonstorm periods: CRRES results, J. Geophys. Res., 115, A12248, doi:10.1029/2010JA015716, 2010.

Hauge, R. and Søraas, F.: Precipitation of $>115 \mathrm{keV}$ protons in the evening and forenoon sectors in relation to the magnetic activity, Planet. Space Sci., 23, 1141-1154, 1975.

Iijima, T., Potemra, T. A., and Zanetti, L. J.: Large-Scale Characteristics of Magnetospheric Equatorial Currents, J. Geophys. Res., 95, 991-999, doi:10.1029/JA095iA02p00991, 1990.

Kennel, C. F. and Petschek, H. E.: Limit on stably trapped particle fluxes, J. Geophys. Res., 71, 1-28, doi:10.1029/JZ071i001p00001, 1966.

Le, G., Russell, C. T., and Takahashi, K.: Morphology of the ring current derived from magnetic field observations, Ann. Geophys., 22, 1267-1295, doi:10.5194/angeo-22-1267-2004, 2004. 
Liemohn, M. W., Kozyra, J. U., Clauer, C. R., and Ridley, A. J.: Computational analysis of the near-Earth magnetospheric current system during two-phase decay storms, J. Geophys. Res., 106, 29531-29542, doi:10.1029/2001JA000045, 2001a.

Liemohn, M. W., Kozyra, J. U., Thomsen, M. F., Roeder, J. L., Lu, G., Borovsky, J. E., and Cayton, T. E.: Dominant role of the asymmetric ring current in producing the stormtime Dst*, J. Geophys. Res., 106, 10883-10904, doi:10.1029/2000JA000326, 2001b.

Lvova, E. A., Sergeev, V. A., and Bagautdinova, G. R.: Statistical study of the proton isotropy boundary, Ann. Geophys., 23, 13111316, doi:10.5194/angeo-23-1311-2005, 2005.

Nakabe, S., Iyemori, T., Sugiura, M., and Slavin, J. A.: A statistical study of the magnetic field structure in the inner magnetosphere, J. Geophys. Res., 102, 17571-17582, doi:10.1029/97JA01181, 1997.

Ohtani, S., Ebihara, Y., and Singer, H. J.: Storm-time magnetic configurations at geosynchronous orbit: Comparison between the main and recovery phases, J. Geophys. Res., 112, A05202, doi:10.1029/2006JA011959, 2007.

Perez, J. D., Grimes, E. W., Goldstein, J., McComas, D. J., Valek, P., and Billor, N.: Evolution of CIR storm on 22 July 2009, J. Geophys. Res., 117, A09221, doi:10.1029/2012JA017572, 2012.

Sergeev, V. A. and Tsyganenko, N.: Energetic particle losses and trapping boundaries as deduced from calculations with a realistic magnetic field model, Planet. Space Sci., 30, 999-007, 1982.

Sergeev, V. A., Malkov, M., and Mursula, K.: Testing the Isotropic Boundary Algorithm Method to Evaluate the Magnetic Field Configuration in the Tail, J. Geophys. Res., 98, 7609-7620, doi:10.1029/92JA02587, 1993.

Sergeev, V. A., Kornilova, T. A., Kornilov, I. A., Angelopoulos, V., Kubyshkina, M. V., Fillingim, M., Nakamura, R., McFadden, J. P., and Larson, D.: Auroral signatures of the plasma injection and dipolarization in the inner magnetosphere, J. Geophys. Res., 115, A02202, doi:10.1029/2009JA014522, 2010.
Sibeck, D. G. and Angelopoulos, V.: THEMIS science objectives and mission phases, Space Sci. Rev., 141, 35-59, doi:10.1007/s11214-008-9393-5, 2008.

Siscoe, G. L. and Crooker, N. U.: On the Partial Ring Current Contribution to Dst, J. Geophys. Res., 79, 1110-1112, doi:10.1029/JA079i007p01110, 1974.

Soraas, F., Lundblad, J. A., and Hultqvist, B.: On the energy dependence of the ring current proton precipitation, Planet. Space Sci., 25, 757-763, 1977.

Søraas, F., Lundblad, J. A., Maltseva, N. F., Troitskaya, V., and Selivanov, V.: A comparison between simultaneous I.P.D.P. groundbased observations and observations of energetic protons obtained by satellites, Planet. Space Sci., 28, 387-405, 1980.

Søraas, F., Aarsnes, K., Oksavik, K., and Evans, D. S.: Ring current intensity estimated from low-altitude proton observations, J. Geophys. Res., 107, 1149, doi:10.1029/2001JA000123, 2002.

Tsyganenko, N. A.: Effects of the solar wind conditions on the global magnetospheric configuration as deduced from data-based field models, in: Proceedings of the Third International Conference on Substorms (ICS-3), Versailles, France, 12-17 May 1996, 181-185, 1996.

Tsyganenko, N. A. and Fairfield, D. H.: Global shape of the magnetotail current sheet as derived from Geotail and Polar data, J. Geophys. Res., 109, A03218, doi:10.1029/2003JA010062, 2004.

Tsyganenko, N. A. and Sitnov, M. I.: Modeling the dynamics of the inner magnetosphere during strong geomagnetic storms, J. Geophys. Res., 110, A03208, doi:10.1029/2004JA010798, 2005.

Tsyganenko, N. A. and Sitnov, M. I.: Magnetospheric configurations from a high-resolution data-based magnetic field model, J. Geophys. Res., 112, A06225, doi:10.1029/2007JA012260, 2007.

Tsyganenko, N. A., Singer, H. J., and Kasper, J. C.: Storm-time distortion of the inner magnetosphere: How severe can it get?, J Geophys. Res., 108, 1209, doi:10.1029/2002JA009808, 2003.

Yahnin, A. G. and Yahnina, T. A.: Energetic proton precipitation related to ion-cyclotron waves, J. Atmos. Solar Terr. Phys., 69, 1690-1706, 2007. 